\title{
Aquagenic Wrinkling of the Palms: Morphological Changes in Reflectance Confocal Microscopy and High-Definition Optical Coherence Tomography
}

\author{
Lea Bielicky Markus Braun-Falco Thomas Ruzicka Tanja Maier \\ Department of Dermatology and Allergology, Ludwig Maximilian University of Munich, Munich, Germany
}

\section{Key Words}

Aquagenic palmoplantar keratoderma .

High-definition optical coherence

tomography · Optical imaging · Reflectance confocal microscopy

\begin{abstract}
Aquagenic wrinkling of the palms (AWP) is a rare condition, which is characterized by appearance of whitish papules and plaques, and an excessive wrinkling and swelling of the palmar skin after exposure to water. In most cases, young women are affected, and an association of AWP with cystic fibrosis (CF) has been surmised. We report on two cases of AWP, which were not related to CF, in whom we used two innovative imaging techniques, namely high-definition optical coherence tomography and reflectance confocal microscopy, to show in vivo skin changes occurring after exposure of the skin to tap water in comparison to the findings in a healthy control person.
\end{abstract}

(C) 2015 S. Karger AG, Basel

\section{Cases}

Two patients presented shortly after each other to our outpatient clinic and reported about similar symptoms.
A 22-year-old female patient (patient 1) reported a 1-year history of a recurrent palmar eruption of whitish papules and an extensive wrinkling of the skin within 23 min after exposure to water. This phenomenon was accompanied by a burning sensation and occasionally moderate pain. The plantar skin was not involved. She had similar symptoms approximately 3 years ago, which vanished without therapy after several months. After symptom recurrence, she had tried a therapy with topically applied steroid cream, which showed no effects.

The 2nd patient (patient 2), a 35-yearold woman, had a 5-year history of similar signs, but in contrast to the 1st patient the skin eruption appeared only on her left palm and on the lateral part of her left foot and was not accompanied by any discomfort or pain.

Both patients were in good health, had no history of hyperhidrosis and were unaware of family members with similar clinical findings. Skin examination revealed whitish well-defined papules on both palms of patient 1 and on the left palm and the lateral part of the left foot of patient 2 as well as prominent eccrine ducts of the sweat glands. Approximately $3 \mathrm{~min}$ after exposure to water, the skin lesions increased impressively in size and the affected skin showed excessive wrinkling and edematous swelling. Roughly 5 min after the water contact, the skin resumed its original state.

We performed punch biopsies in both patients; histology did not reveal any pathological changes. In addition, we initiated a genetic examination in order to find out whether our patients were heterozygous for cystic fibrosis (CF). None of the mutations typical for CF were detected.

In order to visualize the morphological skin changes that take place after the exposure to water in our patients, we performed in vivo reflectance confocal microscopy (RCM) imaging using a commercially available RCM device (Vivascope ${ }^{\circledR}$ 1500; Lucid-Tech Inc., Henrietta, N.Y., USA/ Mavig GmbH, Munich, Germany).

The palms of the 2 patients and 1 gender-matched healthy control person were examined by RCM, performing horizontal mapping (VivaBlock ${ }^{\circledR}, 8 \times 8 \mathrm{~mm}$ ) at the level of the stratum corneum before and 2-5 min after water contact.

Additionally, high-definition (HD) optical coherence tomography (OCT) was performed using a commercially available full-field HD-OCT system (Skintell ${ }^{\circledR}$; Agfa

L. Bielicky and M. Braun-Falco contributed equally to this work.

\section{KARGER 125}

(c) 2015 S. Karger AG, Base

$1018-8665 / 15 / 2303-0208 \$ 39.50 / 0$
PD Dr. med. Tanja Maier

Department of Dermatology and Allergology

Ludwig Maximilian University, Frauenlobstrasse 9-11

DE-80337 Munich (Germany)

E-Mail tanja.maier@med.uni-muenchen.de 

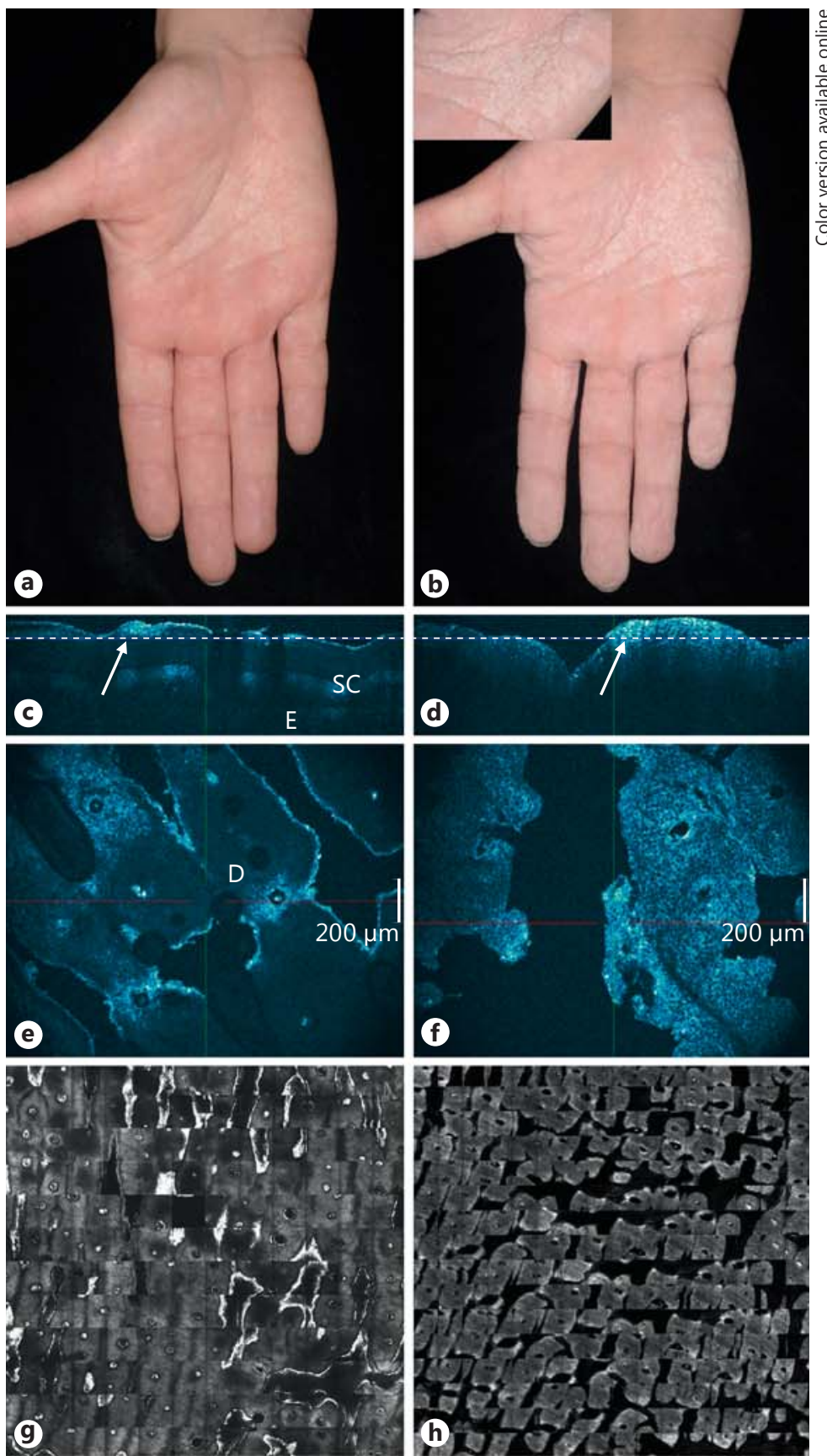

Fig. 1. The right palm of patient 1 shows whitish papules before water contact (a) and palmar wrinkling and swelling 2 min after water contact (b, inset with magnification of the wrinkling). HD-OCT of the palm in the slice mode (c), with typical layering of the stratum corneum (SC) and epidermis (E), and in the en-face mode (e, horizontal image at the level indicated in the dashed line of the vertical image in $\mathbf{c}$, image size $1.8 \times 1.5 \mathrm{~cm}$ ) with ducts of sweat glands (D) at the upper level of the SC. The arrow (c) indicates the irregularity of the SC with hyperreflective areas in the apical part of the SC of the AWP patient, which are absent in the healthy control (see fig. 2). The HD-OCT slice mode (d) and en-face mode (f) of the palm display the broadened, but irregular SC and defined cell borders after water contact. In the RCM, the mosaic $(8 \times 8 \mathrm{~mm})$ displays the irregularity of the sweat gland distribution and disordered alignment of the palmar lines ( $\mathbf{g}$ ) in AWP especially in comparison to the healthy control (see fig. 2) and the swelling after water contact at the upper level of the SC (h). The single RCM image $(0.5 \times 0.5 \mathrm{~mm})$ at the same level depicts the bright thickened and irregular intercellular spaces (i, arrow) which are clearly visible after water contact (j); compared to the healthy control, hyperreflective areas are found (arrow).
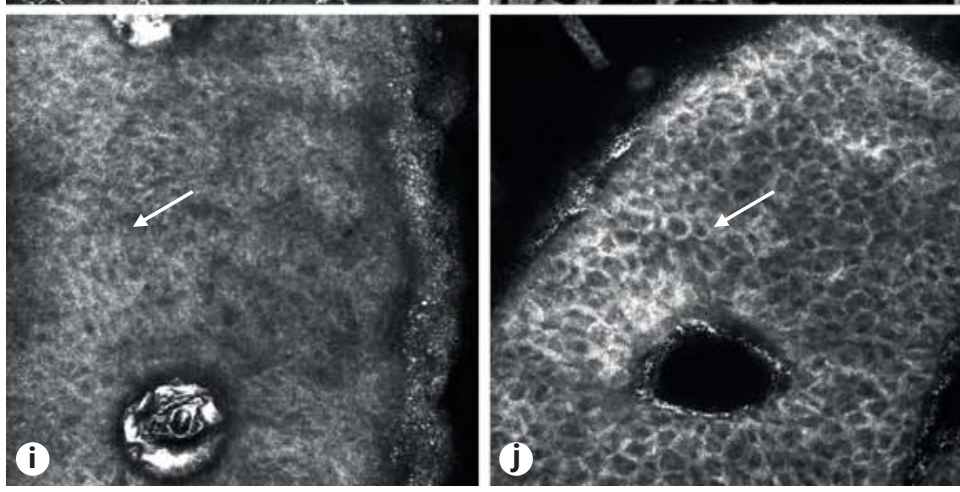
HealthCare GmbH, Brussels, Belgium). It is based on the principle of a 'time domain' OCT system with dynamic focus tracking. According to the producer's instruction, Skintell allows a resolution of $3 \mu \mathrm{m}$ in all three dimensions. In this system, two imaging modes, a vertical (slice) and a horizontal (en face) mode, are available.

The palms of both patients and the healthy control person were evaluated by HD-OCT in the vertical and en-face mode before and 2-5 min after water contact, and images were recorded.

Using HD-OCT, the thickening of the stratum corneum was clearly displayed in the slice mode showing a thickness of the stratum corneum of around $207 \mu \mathrm{m}$ in patient 1 and $220 \mu \mathrm{m}$ in patient 2 before water contact versus $410 \mu \mathrm{m}$ (patient 1) and $450 \mu \mathrm{m}$ (patient 2) after water contact (fig. 1), compared to $221 \mu \mathrm{m}$ before and $321 \mu \mathrm{m}$ after water contact in the healthy control (fig. 2).

The typical layering of the dermis in the slice mode of HD-OCT vanished with the swelling of the stratum corneum. In the en-face mode of HD-OCT, the swelling of the stratum corneum could be visualized showing a clear cell border with dark nuclei surrounded by bright cell borders in comparison with the image before water contact where cell borders were not so clearly depicted. The same finding was present in the RCM image before and after water contact, where the cell borders in the stratum corneum were precisely demarcated after water contact. Compared to the healthy control (fig. 2), the AWP patients clearly showed an irregular superficial layer of the stratum corneum with focal hyperreflective areas, which was not present in the control. In the single RCM image at superficial levels of the stratum corneum, the intercellular borders were clearly broadened and hyperreflective also before water contact (fig. 1cf). In the $8 \times 8 \mathrm{~mm}$ mosaic image of the RCM, the pores of the sweat glands seemed more irregularly distributed com- pared to palms without aquagenic wrinkling and no regular palmar lines could be depicted (fig. 1) compared to the healthy control with regular palmar lines (fig. 2).

Both patients received iontophoretic treatment once daily for 6 weeks without success. The skin manifestations were again monitored by HD-OCT and RCM 1 month after the treatment, but no morphological changes compared to the results before treatment could be found. A treatment attempt with botulinum toxin injections, as reported in the literature, was refused by the patients $[1,2]$.

\section{Discussion}

AWP signs are often described as 'handin-the-bucket sign' [3] because of the characteristic wrinkling and swelling that take place after the patients' hands are immersed in water. AWP (synonyms: aquagenic palmoplantar keratoderma, transient aquagenic palmar hyperwrinkling and aquagenic syringeal acrokeratoderma) was first described by Elliot [4] in 1974 in CF patients. Gild and Clay [5] reported on AWP in $80 \%$ of the investigated CF patients and in $25 \%$ of the carriers of the mutated CF gene. Our 2 patients did not present the relevant $\mathrm{CF}$ gene mutations. However, there is accumulating evidence that AWP also appears independent of CF.

Furthermore, AWP has been associated with the intake of COX-2 inhibitors [6], tobramycin [7] and hyperhidrosis [8], but none of them was present in our 2 patients.

It has been proposed that in patients with $\mathrm{CF}$ and hyperhidrosis, the elevated epidermal sodium content might increase the absorption of water above levels of healthy persons $[9,10]$. In general, it is assumed that in AWP patients structural changes in the stratum corneum result in a malfunction following water contact, and an aberrant expression of AQP5, which belongs to the aquaporin water channel family, was shown in the sweat glands of AWP patients. Additionally, hyperplastic glandular epithelium and enlarged eccrine coils were described [8].

AWP patients may benefit from topical application of $15-20 \%$ aluminum chloride solution $[3,11]$ and local injection of botulinum toxin $[1,2]$, but in our patients a 6-week iontophoretic treatment failed to reduce their symptoms.

Imaging techniques such as OCT and RCM have been applied for various indications, including non-melanoma and melanocytic skin cancer [12-14]. Also, inflammatory reactions such as contact or allergic eczema and infestations with parasites have been studied using these techniques $[15,16]$. These innovative imaging devices enable the noninvasive, in vivo evaluation even of transient skin alterations.

In this case report, we record acute morphological changes of the palmar skin after water contact by HD-OCT and RCM, which were not detectable by standard histological techniques because of their transient nature. We were able to document the significant swelling of the stratum corneum after water contact in both patients compared to a healthy control person, and the irregular broadening and brightening of the intercellular borders could be visualized using both HD-OCT and RCM.

\section{Conclusion}

Innovative imaging devices such as HD-OCT and RCM allow the visualization of transient skin alterations in patients with AWP, which cannot be demonstrated by histological means. In vivo imaging has the additional advantage of repeated noninvasive examination to monitor treatment success.

\section{Disclosure Statement}

T. Maier served as lecturer/consultant for Agfa HealthCare GmbH. There were no funding sources.
Fig. 2. Palm of a healthy control person in HD-OCT with a thin and regular stratum corneum (SC) in the slice mode (a) and regular aligned ducts of sweat glands (D) before water contact in the en-face mode $(\mathbf{c}, 1.8 \times 1.5 \mathrm{~cm})$. After water contact, the $\mathrm{SC}$ shows thickening in the slice mode (b); the horizontal image (en-face mode, $\mathbf{d}$ ) is located in the upper level of the SC, as indicated by the dashed line in the slice-mode image. Arrows indicate the regular
SC with broadening after water contact (b). In the RCM mosaic $(8 \times 8 \mathrm{~mm})$ before $(\mathbf{e})$ and after $(\mathbf{f})$ water contact, the regular palmar lines are depicted at a superficial layer of the SC. The single image $(0.5 \times 0.5 \mathrm{~mm})$ at this level shows fine intercellular spaces (g) compared to the AWP patients which become more prominent after water contact $(\mathbf{h}) . \mathrm{E}=$ Epidermis.

(For figure see next page.) 

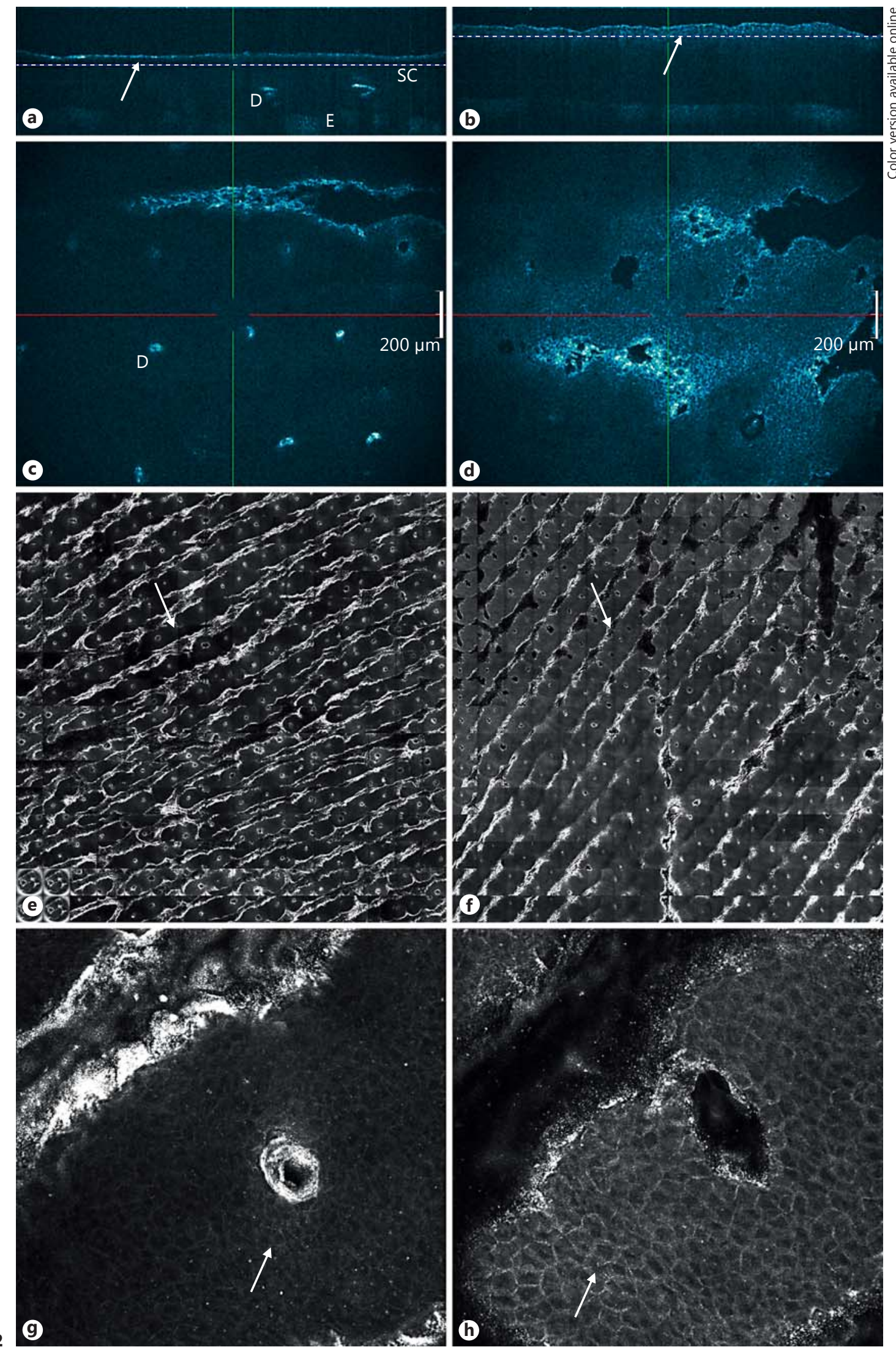


\section{References}

1 Bagazgoitia L, Perez-Carmona L, Salguero I, Harto A, Jaen P: Letter: aquagenic keratoderma: successful treatment with botulinum toxin. Dermatol Surg 2010;36:434-436.

$\checkmark 2$ Diba VC, Cormack GC, Burrows NP: Botulinum toxin is helpful in aquagenic palmoplantar keratoderma. Br J Dermatol 2005;152: 394-395.

-3 Yan AC, Aasi SZ, Alms WJ, James WD, Heymann WR, Paller AS, Honig PJ: Aquagenic palmoplantar keratoderma. J Am Acad Dermatol 2001;44:696-699.

4 Elliott RB: Letter: wrinkling of skin in cystic fibrosis. Lancet 1974; ii: 108.

$\checkmark 5$ Gild R, Clay CD: Aquagenic wrinkling of the palms in a cystic fibrosis carrier. Australas J Dermatol 2008;49:19-20.

$\checkmark 6$ Khuu PT, Duncan KO, Kwan A, Hoyme HE, Bruckner AL: Unilateral aquagenic wrinkling of the palms associated with aspirin intake. Arch Dermatol 2006;142:1661-1662.

$>7$ Ludgate MW, Patel D, Lamb S: Tobramycininduced aquagenic wrinkling of the palms in a patient with cystic fibrosis. Clin Exp Dermatol 2009;34:e75-e77.
8 Kabashima K, Shimauchi T, Kobayashi M, Fukamachi S, Kawakami C, Ogata M, Kabashima R, Mori T, Ota T, Fukushima S, Hara-Chikuma $M$, Tokura Y: Aberrant aquaporin 5 expression in the sweat gland in aquagenic wrinkling of the palms. J Am Acad Dermatol 2008;59(2 suppl 1):S28-S32.

$\checkmark 9$ Tolland JP, Boyle J, Hall V, McKenna KE, Elborn JS: Aquagenic wrinkling of the palms in an adult cystic fibrosis population. Dermatology 2010;221:326-330.

10 Adisen E, Karaca F, Gurer MA: Transient reactive papulotranslucent acrokeratoderma in a 50-year-old woman: case report and review of the literature. Am J Clin Dermatol 2008;9: 404-409.

11 Kocaturk E, Kavala M, Buyukbabani N, Turkoglu Z: Whitish papules on the palm. Int J Dermatol 2007;46:736-737.

12 Maier T, Braun-Falco M, Hinz T, SchmidWendtner MH, Ruzicka T, Berking C: Morphology of basal cell carcinoma in high definition optical coherence tomography: en-face and slice imaging mode, and comparison with histology. J Eur Acad Dermatol Venereol 2013;27:e97-e104.
13 Maier T, Braun-Falco M, Laubender RP, Ruzicka T, Berking C: Actinic keratosis in the en-face and slice imaging mode of high-definition optical coherence tomography and comparison with histology. Br J Dermatol 2013;168:120-128.

14 Maier T, Sattler EC, Braun-Falco M, Korting HC, Ruzicka T, Berking C: Reflectance confocal microscopy in the diagnosis of partially and completely amelanotic melanoma: report on seven cases. J Eur Acad Dermatol Venereol 2013;27:e42-e52.

15 Sattler EC, Maier T, Hoffmann VS, Hegyi J, Ruzicka T, Berking C: Noninvasive in vivo detection and quantification of Demodex mites by confocal laser scanning microscopy. Br J Dermatol 2012;167:1042-1047.

16 Maier T, Sattler E, Braun-Falco M, Ruzicka T, Berking C: High-definition optical coherence tomography for the in vivo detection of Demodex mites. Dermatology 2012;225:271276 